\title{
BMJ Open Developing a collaborative learning framework for resilience in healthcare: a study protocol
}

\author{
Cecilie Haraldseid-Driftland (D) , ${ }^{1}$ Karina Aase (D) , ${ }^{1}$ Siri Wiig (D) , ${ }^{1}$ Stephen Billett ${ }^{2}$
}

To cite: Haraldseid-Driftland C, Aase K, Wiig S, et al. Developing a collaborative learning framework for resilience in healthcare: a study protocol. BMJ Open 2021;11:e045183. doi:10.1136/ bmjopen-2020-045183

- Prepublication history for this paper is available online. To view these files, please visit the journal online (http://dx.doi org/10.1136/bmjopen-2020045183).

Received 24 September 2020 Accepted 28 July 2021

Check for updates

(C) Author(s) (or their employer(s)) 2021. Re-use permitted under CC BY-NC. No commercial re-use. See rights and permissions. Published by BMJ.

${ }^{1}$ SHARE - Centre for Resilience in Healthcare, University of Stavanger, Stavanger, Norway

${ }^{2}$ School of Education and Professional Studies, Griffith University, Mount Gravatt, Queensland, Australia

Correspondence to Dr Cecilie Haraldseid-Driftland; cecilie.haraldseid@uis.no

\section{ABSTRACT}

Introduction Resilience in healthcare (RiH) can be conceptualised as the adaptive capacities of a healthcare system that allow it to maintain the delivery of high-quality care during and after events that challenge, change or disrupt its activities. These adaptive capacities require collaborative learning and working, as the complexities of changes and challenges can rarely be addressed by individuals alone or single healthcare disciplinary knowledge. So, there is a need to understand how collaborative learning practices can be developed and supported both intra and inter disciplinary in healthcare. The aim of the study is to explore the relationship between collaborative learning, and resilience to establish a framework that supports the development and application of adaptive capacities across diverse healthcare contexts and levels. Collaborative learning is premised on learning as something that occurs continuously through everyday work in the healthcare systems as professionals engaging in clinical work, and interacting with other coworkers, patients and stakeholders making local adaptations in respond to needs.

Method and analysis The study applies a mixed methods design in a two-phased approach to explore and develop the relationship between collaborative learning and resilience. Phase One is exploratory using literature review, metasynthesis, interviews and focus groups as data collection methods in empirical studies in different healthcare contexts. Phase Two uses participatory approach to develop and test a collaborative learning framework followed by an evaluation to appraise its utility using observation and focus groups as data collection procedures.

Ethics and dissemination Phase One of the study is approved by the Norwegian Centre for Research Data (reference no. 864334). The findings will be disseminated through scientific articles, presentations at international conferences and through social media and popular press. This includes establishing a set of learning tools for adaptive use, that is made publicly available in Open Access repositories.

\section{INTRODUCTION}

Resilience in healthcare ( $\mathrm{RiH})$ can be conceptualised as the adaptive capacities of a healthcare system that allow it to maintain the delivery of high-quality care during and after events that challenge, change or disrupt its activities, ${ }^{1}$ ranging from high impact situation such as dealing with a pandemic, to everyday

\section{Strengths and limitations of this study}

- The study translates concepts of resilience in healthcare at the system level into practice by developing and testing a collaborative learning framework.

- The study adopts a participatory approach in the coconstruction, development and testing of the collaborative learning framework, involving stakeholders from a range of healthcare contexts and levels.

- The study develops actionable learning tools and principles for improving adaptive capacities in diverse healthcare contexts.

- A 5-year study period might limit the possibilities to document impact of the collaborative learning framework in the longer term.

- Adapting the collaborative learning framework to be applicable in diverse healthcare contexts and levels might create tensions with system imperatives associated with standardisation, creating conflicts with flexibility at the practice level.

challenges such as lack of resources. This includes the individual and collective adaptive capacities of workers in those system. These capacities involve engaging people in collaborative and coordinated processes that adapt, enhance or reorganise work practices or system functioning, where healthcare practices are enacted. ${ }^{2}$ In addition to collaborative and coordinated learning processes, $\mathrm{RiH}$ includes learning from successful practices where highquality care has been maintained despite challenges, change or disruptions. ${ }^{13}$ In the literature, adaptations are linked to learning in theories of resilience ${ }^{45}$ but there is a lack of systematic effort to address the collaborative element of learning processes where healthcare workers, patients and carers adapt and learn together at work. ${ }^{16}$ This knowledge gap is to be addressed in this study.

\section{Collaborative learning and pedagogical rich activities}

Collaborative working and learning have proven ability to effectively respond to changes in practice requirements and developing 
capacities to do so in both communities and workplace ${ }^{78}$ and is held to have advantages over individual learning efforts. ${ }^{9}$ It occurs when individuals collaborate to achieve shared goals and to learn through and from collaborative activities and interactions. ${ }^{10}$ Studies proposing the strengths of collaborative working and learning usually ground their argument in social constructivist theories, as they emphasise learning arising inter-psychologically (ie, between persons and social sources) through interactive learning activities where the knowledge is constructed through interactions ${ }^{11}$ or co-participation. ${ }^{12}$ Key factors for collaborative learning are active social interaction, common group goals and individual accountability. ${ }^{13}$ Some premises for effective collaborative learning are its ability to support the development of shared meaning and practices through interactions among group members, saving time and effort and being informed by the group's joint efforts. ${ }^{14}$ Complex tasks and learning can be effectively mediated by the sharing of the task demands across the groups' membership. This can also provide for more contributions and reduces the need for individual working memory load, thereby making it easier for everyone involved. ${ }^{15}$ In fact, this kind of collaborations are identified as being particularly pedagogically-rich activities. $^{7}$ That is, activities have qualities which inherently promote not only learning per se, but the kinds of learning that are often difficult to secure in other ways. For instance, in mortality and morbidity meetings, handovers and case examinations of unusual outcomes it was found that these activities commonly: (1) provide practitioners access to healthcare knowledge through others' insights and practices; (2) permit comparisons and appraisals with what these practitioners know, can do and value; and (3) offer interactions and access to that knowledge, which might not otherwise be available in practice or education settings. Moreover, these activities can occur intra-professionally or inter-professionally. ${ }^{7}$

\section{Linking collaborative learning and resilience}

Although there could be a number of different ways to address the learning component in $\mathrm{RiH}$, collaborative learning is of particular interest since healthcare provision is based on collaboration among different stakeholders, working and learning together. ${ }^{6}$ Collaborative processes of learning are, therefore, especially linked to resilience through the underlying mechanisms of adaptation which happen when people in systems act on disruptions, challenges and changes. Collaborative learning is premised on learning as something that occurs continuously through everyday work in the healthcare systems as professionals engaging in clinical work, and interacting with other coworkers, patients and stakeholders making local adaptations in response to needs. ${ }^{13}{ }^{16}$ Providing healthcare has increasingly become a shared effort between different stakeholders who work collaboratively to address patients' needs. ${ }^{8}$ This high level of interdependence among healthcare professionals and stakeholders has resulted in evidence suggesting that improved collaborative learning skills, such as communication and coordination skills, can play key roles in improved healthcare performance than a reliance of individual clinical skills. ${ }^{171}$ Improved healthcare provision is, therefore, not just about learning as individuals, but also about learning collaboratively and collectively across stakeholders and system levels.

While the resilience literature has persistently claimed that learning is an essential potential for resilience, ${ }^{19}$ Wiig et $a l^{1}$ propose learning and, in particular, collaborative learning as a prerequisite to operationalise resilience. In the current literature, a few studies address the learning potential viewing it as an outcome resulting in specific adaptive practices to handle capacity-demand misalignments, such as workarounds, ${ }^{20}$ secret second handovers ${ }^{21}$ or next of kin agency. ${ }^{22}{ }^{23}$ Furthermore, resilience studies often focus on individual learning outcomes, such as openness for change, ${ }^{24}$ or people-technology interaction, ${ }^{25}$ with limited focus on team learning or collaborative learning approaches. Studies explicitly describing how learning is related to resilience often highlight a simulation based approach because of its perceived ability to strengthen clinical skills and healthcare personnel's ability to respond to different situations. ${ }^{26-28}$ However, emphasis on collaborative learning covering interactions, pedagogical rich activities and reflexive practice is still limited. ${ }^{1}$

Diverse learning tools are advanced to support development of individual and collaborative learning processes. Simulation-based activities, role play, e-learning, webinars, e-dialogue forums, digital guides and gaming are all examples of such learning tools. In terms of resilience, learning tools hold an untapped potential for advancing the field. However, little is known about which learning approaches and tools that can support and improve $\mathrm{RiH}$. Recently, for instance, the use of video-reflexive ethnography has been adopted whereby video recordings are taken within practice, and then, with appropriate permissions, shared with entire healthcare teams for them to appraise the kinds of actions and interactions occurred, and how these might be enhanced. ${ }^{29-31}$ The concern here is explore how these and other collaborative processes can support RiH.

In sum, most research efforts concerning collaborative learning and resilience have been undertaken in mono disciplinary, single site research concentrating on either resilience or learning. In order to advance to the next step, trying to improve healthcare provision through collaborative learning, the field needs multisite, cross level investigations, trying to operationalise how adaptive capacities can be strengthened through collaborative learning efforts.

\section{Aim and research questions}

This study on developing a collaborative learning framework for $\mathrm{RiH}$ is part of a comprehensive research programme entitled Resilience in Healthcare (RiH) (1 September 2018 to 31 October 2023) that consists of five inter-related work packages (WPs). ${ }^{62}$ The primary 
objective of the overall RiH programme is to reform the understanding of quality in healthcare by development and testing of a theoretical and practical $\mathrm{RiH}$ framework. ${ }^{6}$ This study protocol is, however, limited to the WP concerned with collaborative learning in the RiH programme.

\section{Aim}

The primary aim of this study is to explore how the role of collaborative learning in $\mathrm{RiH}$ can be described and improved. More precisely, the study explores the relationship between collaborative learning and resilience, to develop a framework for collaborative learning that supports adaptive capacities across diverse healthcare settings and system levels. The study is divided into the two main phases: (1) exploration and (2) development and testing. The phases are related to the following main objectives and research questions. The three objectives are to:

1. Explore how the role of collaborative learning is described in relation to resilience

2. Translate RiH into practice by developing a framework for collaborative learning aiming at strengthening adaptive capacities, in different healthcare settings and across levels

3. Evaluate which components and processes are involved in supporting effective collaborative learning in translation of $\mathrm{RiH}$ into practice.

\section{Research questions}

The research questions for this study are:

a. How is the role of collaborative learning described in relation to resilience in the literature?

b. Which pedagogically rich activities can be identified, across different healthcare settings and levels as suitable for collaborative learning?

c. What kinds of collaborative learning needs exist across different healthcare settings and levels?

d. Which collaborative learning components can support the development and strengthening of adaptive capacities across different healthcare settings and levels?

e. How can technology-based collaborative learning tools be developed to strengthen and support adaptive capacities?

f. What are the components and processes involved in supporting effective collaborative learning in translation of RiH into practice in different healthcare contexts and levels?

\section{METHODS AND ANALYSIS \\ Design}

A sequential mixed methods design ${ }^{33}$ using a two-phased approach has been selected to explore and develop the relationship between collaborative learning and resilience. Phase One is exploratory using literature review, metasynthesis of results from a sample of existing empirical studies, interviews and focus groups as main data collection methods. Phase Two uses a participatory approach ${ }^{34}$ to develop and test a collaborative learning framework followed by a process evaluation to study impact using observation and focus groups as data collection methods (see table 1).

Table 1 presents an overview of how these objectives and research questions are aligned with method, sample and data analyses. Together, this table sets out the intentions for and the means through which the investigation will be enacted.

\section{Phase one: exploration}

In the exploratory phase, the key aim is to explore how the role of collaborative learning is described in relation to resilience. This will be done through a dual approach, by conducting a scoping review of the literature and an analysis of results from a sample of empirical projects in Norway. A sample of approximately 20 research projects, postdoctoral and $\mathrm{PhD}$ projects will be selected according to a screening protocol and a Quality and Resilience Trigger Tool (see details in Aase $e t a t^{6}$ ). The sample of empirical projects will be drawn from research performed by researchers at the SHARE - Centre for Resilience in Healthcare (hereafter referred to as: SHARE Research centre) in Norway (between 20102018) and will include projects from a wide range of healthcare settings. SHARE constitutes Norway's largest research group studying quality and safety in healthcare. It was established in 2017, and is a multidisciplinary research centre with about 70 affiliated researchers, including nurses, medical doctors, psychologists, lawyers, engineers and safety scientists. The centre's vision is to become an internationally recognised research centre by reforming the understanding of quality and safety in current healthcare systems through a new and comprehensive RiH framework. The centre conducts research in areas such as interdisciplinary team work, co-production, involvement, telecare, regulation, improvement measures and analysis of healthcare processes. The aim of the screening protocol is to systematically map all former and ongoing projects affiliated with the SHARE Research centre to evaluate possible inclusion. The aim of the Quality and Resilience Trigger Tool is to evaluate possible inclusion of the affiliated projects according to their relevance for quality and resilience. The development of the screening protocol and the trigger tool is based on an iterative process with group consensus in the research team. The sample of projects will then be subject to a metasynthesis of existing findings. This approach will provide an overview and identify different collaborative learning components such as needs, pedagogically rich activities, training methods, where the learning takes place, who is involved in the different collaborative learning situations and how different adaptive capacities are learnt and which type of learning processes are most suitable across different healthcare contexts and levels.

\section{Participants}

Approximately 30-40 researchers who are/has been involved in finished, ongoing or newly started research projects with relevance for collaborative learning and/or 
Table 1 Overview of research phases with belonging objectives, research questions, methods, samples and data analyses

\begin{tabular}{|c|c|c|c|c|c|}
\hline Phase & Objective & Research question & Method & Sample & Data analysis \\
\hline \multirow[t]{4}{*}{ 1) Exploration } & \multirow[t]{4}{*}{$\begin{array}{l}\text { 1) To explore how the } \\
\text { role of collaborative } \\
\text { learning is described in } \\
\text { relation to resilience }\end{array}$} & $\begin{array}{l}\text { a) How is the role of } \\
\text { collaborative learning } \\
\text { described in relation to } \\
\text { resilience in the literature? }\end{array}$ & Scoping review & $\begin{array}{l}\text { Peer-reviewed articles, } \\
\text { grey literature }\end{array}$ & $\begin{array}{l}\text { Qualitative } \\
\text { content analysis }\end{array}$ \\
\hline & & $\begin{array}{l}\text { b) Which pedagogically rich } \\
\text { activities can be identified, } \\
\text { across different healthcare } \\
\text { settings and levels as } \\
\text { suitable for collaborative } \\
\text { learning? }\end{array}$ & Meta-synthesis & $\begin{array}{l}20 \text { empirical studies } \\
\text { on quality and } \\
\text { resilience }\end{array}$ & $\begin{array}{l}\text { Inductive } \\
\text { thematic } \\
\text { analysis of } \\
\text { narratives }\end{array}$ \\
\hline & & \multirow{2}{*}{$\begin{array}{l}\text { c) What kinds of } \\
\text { collaborative learning } \\
\text { needs exist across different } \\
\text { healthcare settings and } \\
\text { levels? }\end{array}$} & $\begin{array}{l}\text { Individual } \\
\text { interviews }\end{array}$ & $10-15$ researchers & \multirow{2}{*}{$\begin{array}{l}\text { Thematic } \\
\text { analysis and } \\
\text { joint display of } \\
\text { data }\end{array}$} \\
\hline & & & $\begin{array}{l}3-4 \text { focus group } \\
\text { interviews }\end{array}$ & $10-15$ researchers & \\
\hline \multirow[t]{2}{*}{$\begin{array}{l}\text { 2) Development } \\
\text { and testing }\end{array}$} & \multirow{2}{*}{$\begin{array}{l}\text { 2) To translate } \mathrm{RiH} \text { into } \\
\text { practice by developing } \\
\text { a framework for } \\
\text { collaborative learning } \\
\text { aiming at strengthening } \\
\text { adaptive capacities, } \\
\text { in different healthcare } \\
\text { settings and across } \\
\text { levels }\end{array}$} & \multirow{2}{*}{$\begin{array}{l}\text { d) Which collaborative } \\
\text { learning components can } \\
\text { support the development } \\
\text { and strengthening } \\
\text { of different adaptive } \\
\text { capacities across different } \\
\text { healthcare settings and } \\
\text { levels? } \\
\text { e) How can technology- } \\
\text { based collaborative } \\
\text { learning tools be developed } \\
\text { to strengthen and support } \\
\text { adaptive capacities? }\end{array}$} & $\begin{array}{l}4-7 \text { workshops } \\
8-10 \text { focus group } \\
\text { interviews } \\
20 \text { hours of } \\
\text { observation }\end{array}$ & $\begin{array}{l}15-20 \text { participants } \\
\text { (competence: } \\
\text { resilience, healthcare, } \\
\text { learning tools, } \\
\text { education and } \\
\text { simulation) }\end{array}$ & \multirow[t]{2}{*}{$\begin{array}{l}\text { Thematic } \\
\text { analysis and } \\
\text { joint display of } \\
\text { data }\end{array}$} \\
\hline & & & & $\begin{array}{l}\text { 15-20 participants } \\
\text { (competence: } \\
\text { clinical experts, } \\
\text { learning experts, } \\
\text { representatives from } \\
\text { different healthcare } \\
\text { settings and levels, } \\
\text { patients and next of } \\
\text { kin representatives) }\end{array}$ & \\
\hline
\end{tabular}

$\mathrm{RiH}$, resilience in healthcare.

resilience will participate. The screening process of projects will be based on publicly available information about the projects. ${ }^{6}$ The researchers involved in these included projects, will be approached and recruited to participate in one or more of the data collection methods listed in the following section.

\section{Data collection}

Four different data collection methods are planned in Phase One. First, a meta-synthesis of results from a sample of existing empirical studies synthesising results related to the link between collaborative learning and resilience. Second, individual interviews with 10-15 researchers concerning potential and existing results, activities and experiences related to collaborative learning and resilience in the planning of and/or executing research projects will be conducted. Third, focus group interviews (three to four interviews with five to seven participants) aiming to create discussions among researchers within a range of themes among others collaborative learning activities, pedagogically rich activities, learning components, learning tools and so on. At last, a set of observations of different types of planned activities in the ongoing projects, such as workshops, stakeholder meetings, co-creation activities and so on will be conducted in order to better understand the linkage between collaborative learning and resilience through practical examples. All interview and observation data will be collected, recorded and transcribed. Data collection performed during COVID-19 pandemic will ensure compliance to infection control protocols and will allow for digital data collection if needed and possible. 


\section{Data analysis}

The data analysis will combine both inductive and deductive approaches. Data from the meta-synthesis of existing empirical studies, individual- and focus group interviews and observations will be analysed using a combined thematic inductive approach and a deductive approach according to a set of four key questions deemed central to understand and operationalise the phenomenon of $\mathrm{RiH}$ in both research and practice. ${ }^{1}$ These questions are formulated as: resilience for what, to what, of what and through what. First, narratives of each included empirical study will be developed according to the four questions.

We will develop a narrative of two to three pages of text for each of the included empirical projects. Second, all 20 project narratives will be subject to the inductive thematic synthesising to identify common themes describing the role of collaborative learning in resilience across healthcare contexts and levels, and the conditions of importance for enabling collaborative learning. This combined approach will ensure that we identify key elements of collaborative learning and adaptive capacity in healthcare.

The total data material in Phase One, including the scoping review, the interview data, the observations and the meta-synthesis will be combined to provide a conceptual backdrop for developing a collaborative learning framework. A procedure of joint display of data ${ }^{33}$ will guide the combination of the three data sources in this analysis.

\section{Phase two: development, testing and evaluation}

The findings from Phase One will feed into Phase Two, in which the study applies participatory approach principles to develop and test a set of collaborative learning components and activities (ie, a collaborative learning framework) to support resilient adaptive capacities. The participatory approach processes will be conducted in collaboration with stakeholders and based on the learning needs and pedagogically-rich activities identified in Phase One. Participatory design is based on the principle of different types of stakeholders collaboratively coming to a consensus of what is important and why, during the design and development of artefacts. ${ }^{34}$ Specifics related to what will be designed and how will therefore be dependent on this process. However, the collaborative learning framework content will cover learning components such as structured meeting arenas, simulation scenarios, interactive digital guides, webinars, e-dialogue forums and so on. The underlying principles of the framework will address issues such as learning goals, participants, readiness, procedures and resources. ${ }^{6}$

This collaborative learning framework development phase will entail two different participatory approach processes where distinct stakeholders (eg, patients, next of kin, training specialists, healthcare professionals, managers, policymakers, regulators) will be involved in an iterative process aiming to operationalise the Phase One findings. In the first process (A) principles will be developed through a consensus process about which basic principles underpin the collaborative learning framework. This will include how to structure different learning processes to strengthen adaptive capacity at individual, team and intra-organisational level. A second participatory approach process (B) will then be initiated to develop a set of prototype collaborative learning scenarios developed for different healthcare settings and levels, to operationalise the principles developed in process A. Both scenario outline, how it is designed and for whom, would be dependent on the consensus made through the participatory design process. The scenarios could be outlined as a description of an everyday situation, where different types of adaptations are needed, followed by reflective questions to be discussed in groups. The collaborative activities could be provided through a digital platform or as physical meetings. The activities could also be designed as a collaboration within or across stakeholder groups and within or across healthcare system levels. The learning scenarios will use a platform of independent, already existing tools, highly adaptable for local context and situations. Prototype scenarios will be pilot-tested in small scale studies in a simulation centre at the University of Stavanger. Based on the results from the pilot testing, the scenarios will be further refined and will constitute the basis for further testing of the collaborative learning framework.

We will test and evaluate the collaborative learning framework in three selected empirical settings in the $\mathrm{RiH}$ project. The first setting focuses on healthcare professionals' individual and team-based adaptive capacities. The second setting focuses on organisational adaptive capacities. The third setting focuses on intraorganisational adaptive capacities. A RiH laboratory will be established to assist these testing activities. The testing will take place over a 12-month period. Potential positive and negative impacts of the collaborative framework will be appraised using a participant observation approach as part of a process evaluation. 63536

\section{Participants}

Participants for participatory approach process A will entail 15-20 selected members with competence in key areas such as: $\mathrm{RiH}$, learning tools, education and simulation. Approximately 15-20 participants will be recruited for the participatory approach process B. The participants will include clinical experts, learning experts, representatives from different healthcare system levels, in addition to patients and next of kin representatives from different settings such as hospitals, homecare, nursing homes, general practitioners and prehospital services.

Participants in the test process will be recruited from the three empirical settings. The participants in this phase will include end users from diverse healthcare contexts and at different levels. Approximately 25-30 participants will be involved in testing and evaluating the collaborative learning framework in the three respective empirical settings. A total of approximately 70-90 participants 
will be part of a thorough testing and of a collaborative learning framework.

\section{Data collection}

The data collection throughout both participatory processes $(\mathrm{A}+\mathrm{B})$ will comprise an iterative process of workshops and focus group interviews. Themes of the workshops will be based on the findings from Phase One, such as collaborative learning needs, pedagogical rich activities, training methods, reflexive spaces, simulation scenario development, patient and stakeholder involvement in collaborative learning, principles and structures for collaborative learning. Approximately four to seven workshops and four to seven focus group interviews with four to eight participants will be conducted for this purpose.

The prototype collaborative learning scenarios will be subjected for further development and testing through a RiH laboratory. The RiH laboratory will be a location for trialling the scenarios, which will be revised through an iterative process with different participants. Data for this purpose will be collected through observation and focus group interviews with the participants. We envision approximately 20 hours of observation and four to six focus group interviews with five to seven participants will be conducted to evaluate the prototypes and revised versions.

Data collection during the process evaluation ${ }^{35}$ of the collaborative learning framework will include observation, focus group interviews and individual interviews. Data will be collected before testing midway through, and at the end of the testing period. Between 10-15 interviews, two to three focus groups with five to seven participants are envisaged for each of the three empirical settings before, during and after test period. In addition, we will observe the testing process to understand the impact of the collaborative learning activities. Key elements for interviews and observation are to uncover functionality of the collaborative learning framework content such as collaborative learning needs, pedagogical rich activities, training methods, reflexive spaces, simulation scenario development, principles and structures for collaborative learning. All these data will be collected, recorded and transcribed. The COVID-19 pandemic will have less impact on the second phase as the population will be vaccinated. But compliance to protocols for infection control and adaptation into digital forums when possible, are a planned risk reduction measures.

\section{Data analysis}

The process evaluation will integrate and mix results from interviews, focus group interviews and observation. The procedure of joint display of data ${ }^{33}$ will be applied to identify how adaptive capacity is learnt and adopted, how teamwork, individual and interorganisational adaptive capacity are enabled through a collaborative learning framework.

\section{Trustworthiness}

Trustworthiness $^{37}$ in the research process will be realised through conducting member checks of the qualitative material. We will furthermore ensure active user involvement in the research process including hired co-researchers to provide practice-based feedback in all research phases. In addition, we will apply researcher triangulation in data collection and analysis which ensures incorporation of different perspectives from nurses, doctors, safety scientist, engineers. This implies a research process taking into account potential alternative explanations, theories and conceptualisation of what is observed and collected from the field. Finally, we will organise a meeting structure with group consensus processes during all data analysis to test and refine our analysis among researchers.

\section{Patient and public involvement}

Patient and stakeholder involvement are integrated throughout all research phases in the $\mathrm{RiH}$ project, from project design, planning, data collection, analysis and publication. A patient citizen representative was involved in the project development and is co-chairing the international expert advisory board in the project. In addition, patients and stakeholders will be included in the study as participants, and co-researchers.

\section{Limitations}

A potential limitation in this study is that the empirical studies are all sampled from one research centre. The aim of strengthening adaptive capacities in different healthcare setting and across levels can also prove challenging due to high levels of local variations, and could be restricted to fewer settings or levels if needed. The study could potentially suffer delays, due to unforeseen events such as the COVID-19 pandemic. In order to respond to these challenges, contingency plans particularly related to data collection and recruitment of participants from the healthcare setting have been developed. If necessitated by social distancing requirements, data collection and the participatory design process will be carried out through digital platforms. In addition, the collaborative learning components and activities will be developed and customised to fit a strictly digital format, so that potential restriction related to COVID-19 could be maintained at any particular time.

\section{ETHICS AND DISSEMINATION \\ Ethics}

Phase One of the study is approved by the Norwegian Centre for Research Data (NSD), reference no.: 864334, through the overall $\mathrm{RiH}$ project of which this study is a part of. Ethical approval from the Norwegian Centre for Research Data or the Regional Committees for Medical and Health Research Ethic will be applied for Phase Two pending completion of Phase One. 


\section{Dissemination}

The findings will be disseminated through scientific articles, presentations at national and international conferences and in social media, newsletters, and in the press. Annual stakeholder seminars will contribute to create different collaborative learning arenas involving relevant stakeholders in the development of different new and innovative research. The findings from this study will be used to generate a set of learning tools for adaptive capacities with belonging principles and approaches made available in an Open Access repository. Moreover, the study will build new knowledge on participatory approach involving patients and other stakeholders in the development of a collaborative learning framework.

Contributors Authors KA and $\mathrm{CH}-\mathrm{D}$ advanced the initial idea for the protocol. $\mathrm{CH}-$ $\mathrm{D}, \mathrm{SB}$ and KA drafted the manuscript with major contribution from SW. All authors have commented on the draft, read and approved the final version.

Funding This work was supported by the Norwegian Research council (NFR) grant number 275367. The University of Stavanger, Norway, the Norwegian University of Science and Technology in Gjøvik, and the Norwegian Air Ambulance Foundation support the programme with in-kind funding.

Competing interests None declared.

Patient and public involvement Patients and/or the public were involved in the design, or conduct, or reporting, or dissemination plans of this research. Refer to the Methods section for further details.

Patient consent for publication Not required.

Provenance and peer review Not commissioned; externally peer reviewed.

Open access This is an open access article distributed in accordance with the Creative Commons Attribution Non Commercial (CC BY-NC 4.0) license, which permits others to distribute, remix, adapt, build upon this work non-commercially, and license their derivative works on different terms, provided the original work is properly cited, appropriate credit is given, any changes made indicated, and the use is non-commercial. See: http://creativecommons.org/licenses/by-nc/4.0/.

\section{ORCID iDs}

Cecilie Haraldseid-Driftland http://orcid.org/0000-0002-4085-1972

Karina Aase http://orcid.org/0000-0002-5363-5152

Siri Wiig http://orcid.org/0000-0003-0186-038X

\section{REFERENCES}

1 Wiig S, Aase K, Billett S, et al. Defining the boundaries and operational concepts of resilience in the resilience in healthcare research program. BMC Health Serv Res 2020;20:330.

2 Wiig S, Fahlbruch B. Exploring resilience a scientific journey from practice to theory. Springer Open, 2018.

3 Anderson JE, Ross AJ, Macrae C, et al. Defining adaptive capacity in healthcare: a new framework for researching resilient performance. Appl Ergon 2020;87:103111.

4 Hollnagel E, Braithwaite J, Wears R. Resilient health care. Boca Raton: CRC Press, 2013.

5 Wildavsky A. Searching for safety. New Brunskick: Transaction publishers, 1991.

6 Aase K, Guise V, Billett S, et al. Resilience in healthcare $(\mathrm{RiH})$ : a longitudinal research programme protocol. BMJ Open 2020;10:e038779.

7 Billett S, Noble C, Sweet L. Pedagogically-rich activities in hospital work: Handovers, ward rounds and team meetings. In: Delany $\mathrm{C}$, Molloy L, eds. A practical guide for learning and teaching in a clinical context. Elsevier. Australia, 2018: 207-20.

8 Billett SR. Securing intersubjectivity through interprofessional workplace learning experiences. J Interprof Care 2014;28:206-11.

9 Barron B. Achieving coordination in collaborative problem-solving groups. J Learn Sci 2000;9:403-36.
10 Retnowati E, Ayres P, Sweller J. Can collaborative learning improve the effectiveness of worked examples in learning mathematics? J Educ Psychol 2017;109:666-79.

11 Schreiber LM, Valle BE. Social constructivist teaching strategies in the small group classroom. Small Group Research 2013;44:395-411.

12 Billett S. Toward a workplace pedagogy: guidance, participation, and engagement. Adult Education Quarterly 2002;53:27-43.

13 Billett S. Learning through health care work: premises, contributions and practices. Med Educ 2016;50:124-31.

14 Plass JL, O'Keefe PA, Homer BD, et al. The impact of individual, competitive, and collaborative mathematics game play on learning, performance, and motivation. J Educ Psychol 2013;105:1050-66.

15 Kirschner F, Paas F, Kirschner PA. A cognitive load approach to collaborative learning: United brains for complex tasks. Educ Psychol Rev 2009;21:31-42.

16 Wiig S, Aase K, Bal R. Reflexive spaces: Leveraging resilience into healthcare regulation and management. J Patient Saf 2020. doi:10.1097/PTS.0000000000000658. [Epub ahead of print: 31 Jan 2020].

17 Manser T. Teamwork and patient safety in dynamic domains of healthcare: a review of the literature. Acta Anaesthesiol Scand 2009;53:143-51.

18 Reeves S, Goldman J, Burton A, et al. Synthesis of systematic review evidence of interprofessional education. J Allied Health 2010;39 Suppl 1:198-203.

19 Safety-I HE. Safety-II: the past and future of safety management. Ashgate: Farnham, 2018.

20 Chuang S, Hollnagel E. Challenges in implementing resilient health care. resilient health care: ROUTLEDGE in association with GSE research, 2017: 47-59.

21 Sujan MA, Spurgeon P, Cooke MW. Translating tensions into safe practices through dynamic trade-offs: the secret second handover. resilient health care, volume 2. CRC Press, 2017: 41-52.

22 Bergerød IJ, Braut GS, Wiig S. Resilience from a Stakeholder perspective: the role of next of kin in cancer care. J Patient Saf 2020;16:e205-10.

23 Dyrstad DN, Laugaland KA, Storm M. An observational study of older patients' participation in hospital admission and discharge--exploring patient and next of kin perspectives. J Clin Nurs 2015;24:1693-706.

24 Sheps S, Cardiff K, Pelletier E. Revealing resilience through critical incident narratives: a way to move from Safety-I to Safety-II. Resilient Health Care 2015;2:189-206.

25 Nakajima K. Blood transfusion with health information technology in emergency settings from a Safety-Il perspective. resilient health care. CRC Press, 2017: 129-44.

26 Patterson M, Deutsch ES, Jacobson L. Simulation: closing the gap between work-as-imagined and work-as-done. resilient health care: ROUTLEDGE in association with GSE research, 2017: 143-52.

27 Nakajima S, Masuda S, Nakajima K. Exploring ways to capture and facilitate work-as-done that interact with health information technology. resilient health care. CRC Press, 2016: 82-90.

28 Dieckmann P, Patterson M, Lahlou S, et al. Variation and adaptation: learning from success in patient safety-oriented simulation training. Adv Simul 2017;2:21.

29 Noble C, Billett S, Hilder J, et al. Enriching medical trainees' learning through practice: a video reflexive ethnography study protocol. BMJ Open 2019;9:e031577-9.

30 Mesman J, Walsh K, Kinsman L, et al. Blending Video-Reflexive ethnography with Solution-Focused approach: a Strengths-Based approach to practice improvement in health care. Int J Qual Methods 2019;18:160940691987527.

31 Ajjawi R, Hilder J, Noble C, et al. Using video-reflexive ethnography to understand complexity and change practice. Med Educ 2020;54:1-7.

32 Anderson JE, Aase K, Bal R, et al. Multilevel influences on resilient healthcare in six countries: an international comparative study protocol. BMJ Open 2020;10:e039158.

33 Creswell JW. A Concise introduction to mixed methods research. SAGE publications, 2014.

34 Sanoff H. Multiple views on participatory design. Int J Archit Res 2008;2:57-69.

35 Moore GF, Audrey S, Barker M, et al. Process evaluation of complex interventions: medical Research Council guidance. BMJ 2015;350:h1258.

36 DeWalt KM, DeWalt BR. Participant observation: a guide for fieldworkers. Lanham, Md.: AltaMira Press, 2011.

37 Lincoln YS, Guba EG, Pilotta JJ. Naturalistic inquiry. California: Sage publication, 1985: 438-9. 